Research Paper

\title{
Trends in Alzheimer's Disease Research Based upon Machine Learning Analysis of PubMed Abstracts
}

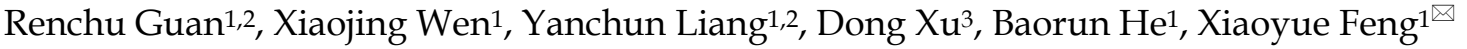 \\ 1. Key Laboratory of Symbolic Computation and Knowledge Engineering of the Ministry of Education, College of Computer Science and Technology, Jilin \\ University, 130012, Changchun, China \\ 2. Zhuhai Sub Laboratory, Key Laboratory of Symbolic Computation and Knowledge Engineering of the Ministry of Education, Zhuhai College of Jilin \\ University, 519041, Zhuhai, China \\ 3. Department of Electric Engineering and Computer Science, and Christopher S. Bond Life Sciences Center, University of Missouri, 65201, Columbia, USA \\ $\square$ Corresponding author: E-mail: fengxy@jlu.edu.cn (Feng, XY).
}

() The author(s). This is an open access article distributed under the terms of the Creative Commons Attribution License (https://creativecommons.org/licenses/by/4.0/). See http://ivyspring.com/terms for full terms and conditions.

Received: 2019.04.15; Accepted: 2019.06.09; Published: 2019.08.06

\begin{abstract}
About 29.8 million people worldwide had been diagnosed with Alzheimer's disease (AD) in 2015, and the number is projected to triple by 2050. In 2018, AD was the fifth leading cause of death in Americans with 65 years of age or older, but the progress of $A D$ drug research is very limited. It is helpful to identify the key factors and research trends of $A D$ for guiding further more effective studies. We proposed a framework named as LDAP, which combined the latent Dirichlet allocation model and affinity propagation algorithm to extract research topics from 95,876 AD-related papers published from 2007 to 2016. Trends and hotspots analyses were performed on LDAP results. We found that the focus points of $A D$ research for the past 10 years include 15 diseases, 15 amino acids, peptides, and proteins, 9 enzymes and coenzymes, 7 hormones, 7 carbohydrates, 5 lipids, 2 organophosphonates, 18 chemicals, 11 compounds, 13 symptoms, and 20 phenomena. Our LDAP framework allowed us to trace the evolution of research trends and the most popular areas of interest (hotspots) on disease, protein, symptom, and phenomena. Meanwhile, 556 AD related-genes were identified, which are enriched in 12 KEGG pathways including the AD pathway and nitrogen metabolism pathway. Our results are freely available at https://www.keaml.cn/Alzheimer.
\end{abstract}

Key words: Alzheimer's disease; Latent Dirichlet Allocation; Affinity Propagation

\section{Introduction}

Between 2010 and 2015, the number of Alzheimer's disease (AD) cases has more than tripled, and the prevalence of AD is still increasing [1,2]. 2015 report estimated that 29.8 million people had been diagnosed with $\mathrm{AD}[3,4]$. As a chronic neurodegenerative disease, $\mathrm{AD}$ is the cause of $60 \%$ to $70 \%$ of dementia cases and in 2015, the G8 nations endorsed $\mathrm{AD}$ as a major societal concern when it was reported as the cause of about 1.9 million deaths [5-9]. Moreover, the number of global AD cases is projected to more than triple by $2050[2,10,11]$. In 2018, AD became the sixth leading cause of death in the United States and the fifth leading cause of death in Americans with 65 years of age or older [12]. It is predicted that by 2050, the number of deaths caused by $\mathrm{AD}$ will account for $43 \%$ of all deaths among the elderly in the United States [4].

$\mathrm{AD}$ is a type of brain disease that begins primarily at the age of 65 or older, although $4 \%$ to $5 \%$ of $\mathrm{AD}$ cases are early-onset [13]. The early symptom in $\mathrm{AD}$ is difficult in remembering recent events, also called short-term memory loss [6,14]. Symptoms arise because part of the brain's nerve cells (neurons) that are involved in thinking, learning, and memory (cognitive function) have been damaged or destroyed[15]. As the disease advances, symptoms may include problems with language, disorientation, and some pathological signs and symptoms such as 
atrophy and paralysis. As an AD sufferer's physical condition declines, much of his or her basic bodily functions are gradually lost, and this will ultimately lead to death [16]. Although the health situation may vary after diagnosis, the life expectancy is 3 to 9 years on average $[17,18]$.

Many journals, conferences, patents, and associations are dedicated to the study of AD. For example, Alzheimer's Association releases an annual report, which describes the public health impact of $\mathrm{AD}$, including incidence and prevalence, mortality and morbidity, use and costs of care, and the overall impact on caregivers and society [19]. There are nearly 0.6 million authorized patents about AD. With the fast development of biotechnology and medicine, a huge amount of biomedical literature and data have been published. For example, until February 2019, 142,785 papers have been published on AD in PubMed. From these research, we can find the research hotspots and trends of $\mathrm{AD}$.

For the curation research on $\mathrm{AD}$, the whole world is struggling to find more effective ways to treat the disease, delay its onset and prevent its development. However, this progress is not satisfactory. For example, it is reported that researchers had spent more than 100 billion dollars to find effective drugs to cure AD in clinical treatments in past years. But the results were disappointed as in the failure of the antibody, solanezumab, which held the promise of improving cognition but failed trial tests [20]. Alzheimer's disease is currently incurable, but treatments for symptoms are available. Although current Alzheimer's treatments cannot stop the disease progressing, they can temporarily slow the deterioration of dementia symptoms and improve the quality of life [14,21]. In 2018, it is reported that more than 16 million family members and other unpaid caregivers provided approximately 18.5 billion hours of care for people with Alzheimer's or other dementia. The value of this care is close to $\$ 234$ billion [15]. AD is considered as one of the most financially costly diseases in developed countries [22,23].

For clinicians or biological researchers, rapid and effective acquisition of cutting-edge information on research advances from tens of thousands publications is a huge challenge [24]. However, because of the complexity of the multi-disciplinary study, there is no research to summarize these findings to keep pace with the research trends of $\mathrm{AD}$ using machine learning and natural language processing models. To address this problem, we adopted machine learning methods to automatically summarize the trends and hotspots of AD based on PubMed abstracts. For this purpose, we collected 10 years of abstracts on AD research from PubMed. Then we proposed a framework named as LDAP which combined latent Dirichlet allocation model and the affinity propagation algorithm to extract research topics and hotspots. By incorporating the Medical Subject Headings term categories, we studied the trends includes medical entity changes in diseases, chemicals and drugs, symptoms and phenomena, and gene pathway based on the hotspots for the past 10 years. There may be other trends changed about AD, but in this study, we only focus on the changes in the areas mentioned above.

\section{Methods}

\section{LDA topic model}

Latent Dirichlet allocation (LDA) is a topic model algorithm based on probability proposed by Blei et al. in 2003 [25]. It is an unsupervised machine learning technique and can be used to identify potentially hidden topic information in large-scale document sets or corpora [26]. LDA is a document theme generation model, which also known as a three-layer Bayesian probability model. It assumes that each word is extracted from a hidden theme behind it, and it contains three layers of words, topics, and documents $[27,28]$. LDA assembles a collection of documents, where $D=\left\{d_{m}\right\}, m \in\{1, \ldots, M\}$. The distribution of topic $k$ over vocabulary is denoted as $\Phi=\left\{\phi^{k}\right\}, k \in\{1, \ldots, K\}$, and the distribution of the $m$-th document over all $K$ topics is denoted as $\Theta=\left\{\theta_{m}\right\}$, $m \in\{1, \ldots, M\}$. A topic is a distribution of terms over a vocabulary. It allows each document to be described as a distribution over topics, which can be expressed as:

$$
\mathrm{P}(w \mid d)=\mathrm{P}(w \mid t) \times \mathrm{P}(t \mid d)(1)
$$

where $w$ represents a word, $d$ represents a document, and $t$ is the topic. It can be expanded as follows:

$$
p\left(\mathrm{w}, \mathrm{z}, \theta_{m}, \Phi \mid \alpha, \beta\right)=p(\Phi \mid \beta) p\left(\theta_{m} \mid \alpha\right) p\left(z \mid \theta_{m}\right) p(\mathrm{w} \mid \Phi, \mathrm{z})
$$

where, for document $m$, the distribution of document over topics $\theta_{m}$ and the distribution of topics over vocabulary $\Phi$ are sampled from priors $a$ and $\beta$, respectively. Then, the topic assignment $z$ for each word is generated from $\theta_{m}$, and the accurate words $w$ are generated according to their respective topic assignment $z$ and the distribution of topics over the vocabulary $\Phi$.

To get the topic words of each year, we used Gibbs sampling to estimate the LDA model. Gibbs sampling is a Markov chain Monte Carlo (MCMC) algorithm for obtaining a sequence of observations which are approximated from a specified multivariate probability distribution, when direct sampling is difficult. 


\section{Affinity Propagation model}

Affinity propagation (AP) is a clustering algorithm based on the concept of "message passing" between data points. AP finds "exemplars", which are members of the input set representing clusters [29]. $\mathrm{AP}$ has been used in many fields, such as image processing [30], text clustering [31], and gene detecting [32].The description of an AP algorithm is as follows: Given a sample set $X=\left\{x_{1}, x_{2}, \ldots, x_{n}\right\}$, and there is no hypothesis of inherent structure between data. Let $\mathbf{S}$ be a matrix that depicts the similarity between points, $s(i, j)>s(i, k)$ if and only if the similarity between $x_{i}$ and $x_{j}$ is greater than that of $x_{i}$ and $x_{k}$. The similarity is represented by the reciprocal of cosine similarity corresponding to (3):

$$
\frac{1}{\cos \theta}=\frac{\sqrt{\sum_{k=1}^{n} x_{i k}^{2}} \cdot \sqrt{\sum_{k=1}^{n} x_{j k}^{2}}}{\sum_{k=1}^{n}\left(x_{i k} \cdot x_{j k}\right)}(3)
$$

The AP algorithm alternates between two messages passing steps to update two matrices:

(a) The responsibility matrix $\mathbf{R}: r(i, k)$ describes the extent to which sample $k$ is suitable for the clustering center of sample $i$, and it represents the messages from $i$ to $k$.

(b) The availability matrix A: $a(i, k)$ describes the selection sample $i$ choosing sample $k$ as the degree of suitability for the cluster center, and it represents the messages from $k$ to $i$.

The matrix $\mathbf{R}$ will be constantly updated according to (4).

$$
r_{t+1}(i, k)=s(i, k)-\max _{k^{\prime} \neq k}\left\{a_{t}\left(i, k^{\prime}\right)+s\left(i, k^{\prime}\right)\right\}
$$

The matrix $\mathbf{A}$ will be continually updated according to (5) and (6).

$$
\begin{gathered}
a_{t+1}(i, k)=\min \left(0, r_{t}(k, t)+\sum_{i^{\prime} \notin(i, k)} \max \left\{0, r_{t}\left(i^{\prime}, k\right)\right\}\right) \\
i \neq k(5) \\
a_{t+1}(k, k)=\sum_{i^{\prime} \neq k} \max \left\{0, r_{t}\left(i^{\prime}, k\right)\right\}(6)
\end{gathered}
$$

After copious iterations, the final clustering result can be obtained when the two matrices converge. To avoid numerical oscillations, a damping factor $\lambda \in(0,1)$ is introduced when updating the two matrices, as described in (7) and (8):

$$
\begin{aligned}
r_{t+1}(i, k) & \leftarrow(1-\lambda) r_{t+1}(i, k)+\lambda r_{t}(i, k)(7) \\
a_{t+1}(i, k) & \leftarrow(1-\lambda) a_{t+1}(i, k)+\lambda a_{t}(i, k)(8)
\end{aligned}
$$

\section{Proposed framework}

This paper presents an LDAP framework, which combines LDA model and AP algorithm to extract research topics. Our proposed framework is depicted in Figure 1. It consists of data processing, methods, and trends analyses. The data from PubMed are downloaded and pre-processed with operations of word segmentation, lemmatization, and stop words removal techniques. After data acquisition and preprocessing, the data is fed to the topics model LDA. The evaluation index, perplexity, in the LDA model can cause some variation in the number of topics. When the number is too small, the model may not reach convergence and achieve the optimal result; meanwhile, the model will lose the ability of capturing topic-diversity and this can cause highly used words like ad, disease, alzheimer, and dementia to get high scores in each topic. Therefore, we generated topics using LDA and then clustered them with AP. With the introduction of MeSH, David, and KEGG, the results can be represented from different aspects, such as proteins, diseases, and pathways.

\section{Experiments and Results}

\section{Data set and preprocessing}

Because an abstract can provide a concise and accurate description of the important content of a paper, necessary information can be obtained from abstracts without reading the full text. We took "Alzheimer's disease" as the entry term to search papers in PubMed and obtained 95,876 papers from 2007 to 2016. Each file is a semi-structured XML document and contains various tags, such as <title>, $<$ abstract $>$, <pmid $>$, etc. We extracted the content in $<$ abstract $>$ and $<$ pmid $>$ fields from the raw XML files. PMID is the unique ID for a paper in PubMed. After extraction, each abstract was stored in a corresponding file.

We obtained statistics in which the number of papers on $\mathrm{AD}$ increased over time. Figure 2 shows that with the aging of society and understanding of $\mathrm{AD}$, more and more research is devoted to the study of $\mathrm{AD}$.

Because the original data contains irrelevant and noisy information that affected the correctness of the final experimental results, a pre-processing operation was necessary to assure the accuracy of the dataset. In our work, the natural language processing techniques such as word segmentation, lemmatization, and stop words removal were applied to the raw data.

\section{Parameters}

In our experiment, for LDA model, we set the topic number $t=200$, hyper-parameters $a=0.25, \beta=0.01$, and the iteration $i=400$ by perplexity evaluation. In the $\mathrm{AP}$ algorithm, we set the damping factor to 0.95 after several adjustments. 


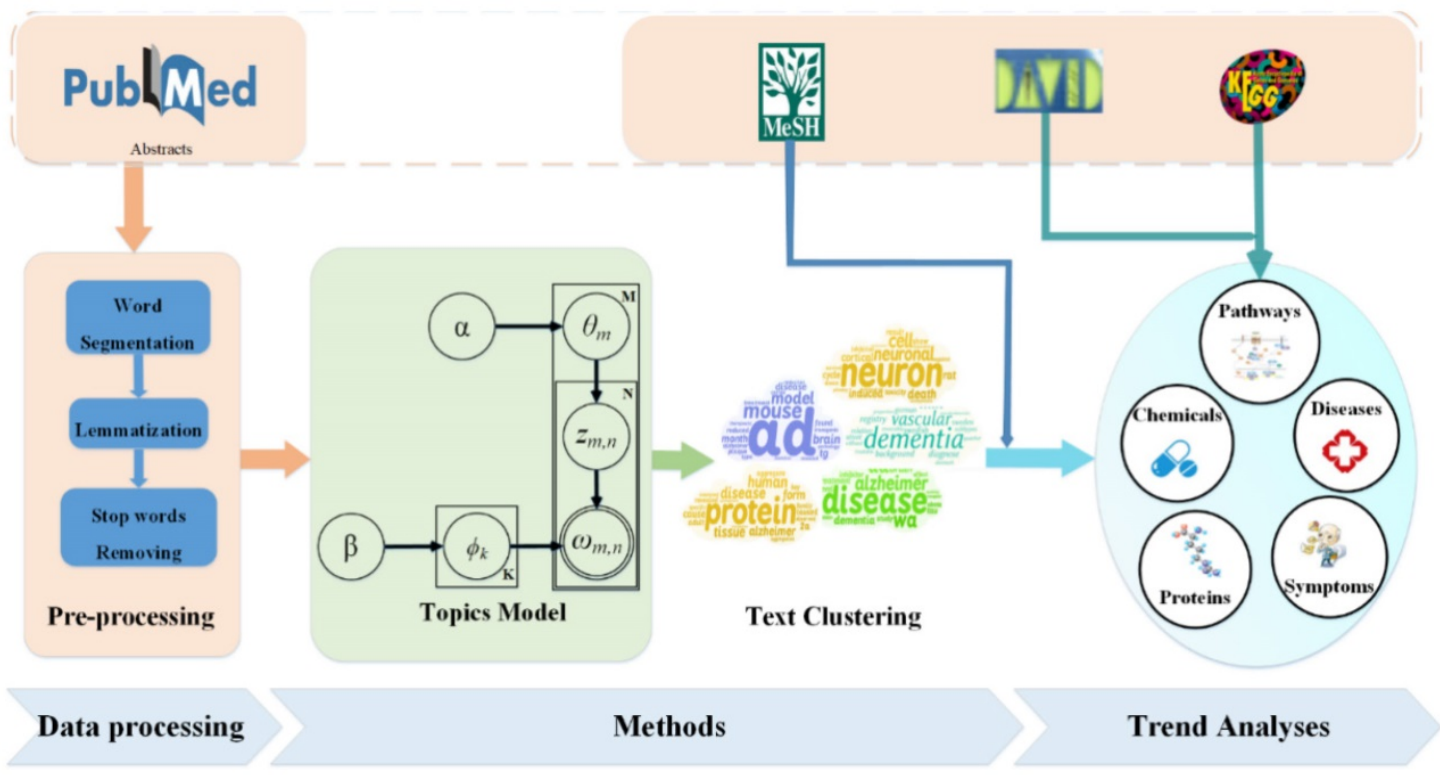

Figure 1. Proposed framework. This framework consists of data processing, methods, and trends analyses. Data downloaded from PubMed was pre-processed and fed into an LDA model. AP algorithm was used to cluster the topics generated from LDA. Trend analyses were done on these topic words.

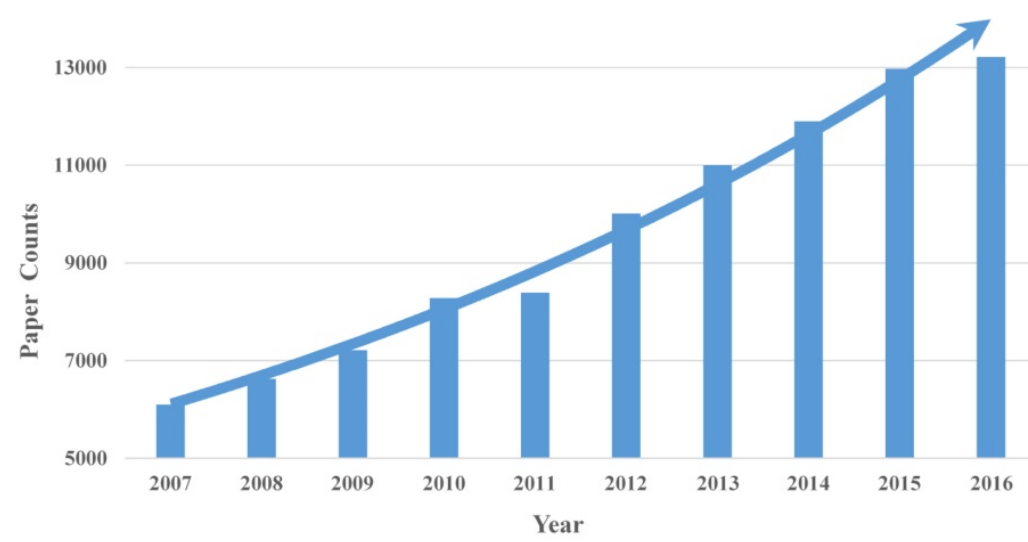

Figure 2. Paper counts on AD from 2007 to 2016. The number of papers on AD increased over time, and the total number of ten years is $95,876$.

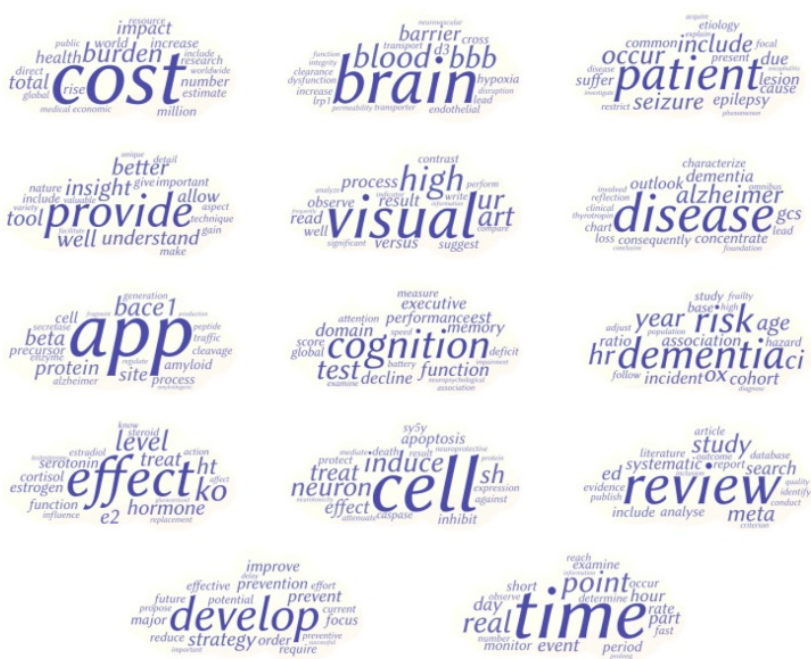

Figure 3. AD research topics of 2016. LDAP results contain 14 clusters and each cluster is represented by 20 words. Word cloud displays different size words. The more weight given a word, the more prominent it is in AD terminology.

\section{Topic results}

Our LDAP framework adopts the LDA model to process the annual data, and 200 topics are obtained for each year. Then the AP algorithm is employed to cluster the LDA results. We used word clouds to visualize the LDAP results. Word cloud displays different size words where the higher the word weight, the bigger the word. Taking the results of 2016 as an example, as shown in Figure 3, the LDAP framework generated 14 clusters, and the central topic words are summarized as "patient", "cost", "develop", "time", "review", "cell", "effect", "provide", "app", "visual", "brain", "cognition", "dementia", and "disease".

In Figure 3, cost is the central word of the first cluster, and the other obvious words such as burden, health, and million are also associated with cost. The caring for persons with AD has caught great attention. $\mathrm{AD}$ is considered as one of the most financially costly 
diseases in developed countries. Moreover, App (Amyloid Precursor Protein) is another central word that can present the whole cluster of protein topics, including beta amyloid protein and bace1 which are directly associated with AD. More research topics from 2007 to 2015 are shown in Supplemental Figure S1-S9.

The topic centers (most used AD terminology, i.e., words associated with hotspots) are presented by years and listed in Table 1. The word after each forward slash (/) is the central word of the biomedical aspect implied in the result, and it also appears in the results at the same time. Some topic-center words such as brain, dementia, cognition, and protein, appear in most of the years and are well-known AD research hotspots. On the contrary, the topic center word, education, appeared only once in 2015. We found a hotspot on the link between education and dementia in 2015 [33].

There are 142 clusters for all ten years' data and 20 words in each central topic. We summed up all the words in the central topics and obtained 1,988 unique words. Then we introduced the Mesh terms to classify the categories of these words, and obtained 75 categories in all, which are: 15 diseases, 15 kinds of amino acids, peptides, and proteins, 9 enzymes and coenzymes, 7 hormones, 7 carbohydrates, 5 lipids, 2 organophosphonates, 18 chemicals, 11 types of compounds, 13 different symptoms, 20 different phenomena, and other categories. In our experiments, we focused our research on 11 categories and recorded the trends and hotspots that the center topics words represent. In the following section, we analyzed trends and hotspots on terminology pertaining to diseases, proteins, symptoms and phenomena, and gene pathways.

\section{Disease hotspots}

Figure 4 illustrates 15 diseases or disorders related to AD. They are amyloidosis, atherosclerosis, hypertension, stroke, diabetes, hypersensitivity, aphasia, encephalitis, encephalopathy, epilepsy, paralysis, Parkinson's, seizures, neurotoxicity and vesicle, which can be categorized into six types of diseases and one type of disorder. For example, amyloidosis belongs to nutritional and metabolic disease, aphasia and Parkinson's are nervous system diseases.

Figure 4 shows that neurotoxicity had appeared 5 times in ten years. Neurotoxicity is a form of toxicity that adversely affects the nervous system and was reported as having a strong relation with Alzheimer's disease. Besides neurotoxicity, some diseases appeared in the same year; for example, aphasia and paralysis appeared in 2010 and 2012, and epilepsy, seizures, and encephalopathy or encephalitis appeared in 2008 and 2016. Aphasia and paralysis are usually associated with symptoms after a stroke. Epilepsy and encephalopathy can damage the nerves of brain. This suggests that these diseases are not only related to Alzheimer's disease, but also have relations among themselves.

For the 15 diseases identified from the hotspots on Alzheimer's disease, we downloaded the abstracts for each disease from 2007 to 2016 to double check the relation between $\mathrm{AD}$ and these diseases. We used the same model to process these 15 diseases to find whether AD appeared in the results. The result is shown in Figure 5. It shows that Alzheimer's disease also appeared in the hotspots of these 15 diseases. Besides, the association between alzheimer's disease and diabetes can be found in the research $[34,35]$. This finding may be helpful in the early diagnosis of $\mathrm{AD}$ and these diseases.

\section{Chemicals and drugs hotspots}

Besides diseases, other entities related to Alzheimer's disease are also found in the results, such as enzymes, hormones, and lipids. The distribution of proteins in each year is shown in Figure 6 . The distribution of other categories in each year is shown in Supplemental Table S1-S3.

Table 1. Topic centers of each year

Years Topic Centers

2007 app; brain; cognition; disease; neuron; treat; dementia; affect; bind; study; effect; problem; study/gender; post; test

2008 protein; cognition; metabolic/disease; human/brain; cost; inhibit; molecule; mechanism; measure; datum; patient; rat; association; study; neurological

2009 microglia; protein; cognition; disease; inhibit; diagnose; method; study; population; provide; domain; significant; region; increase

2010 rat/memory; signal/protein; image/brain; increase; progressive/dementia; synaptic/neuron; diagnose; fibril; progressive; specific; concentration; term; therapy; measure

2011 cell; regulate/brain; cognition; disease; compound/inhibit; clinic; dementia; structure; affect; identify; case; cross; time

2012 amyloid; brain; test/cognition; disease; progressive/syndrome; mechanism; age; method; review; increase; risk; induce; month

2013 app; protein; function/cognition; metabolic; disease; dementia; identify; cortical; effect; research; volume; patient; study; develop; age; show

2014 cell; secretase; function/cognition; injury/brain; disease; evidence; dementia; year; gene; increase; predict; protein; study; control

2015 pet/amyloid; protein; rat/memory; dementia; hypertension; research; study; daily; treat; diagnose; education; progress; disease

2016 cell; app; cognition; patient; brain; cost; develop; time; review; effect; provide; visual; dementia; disease 


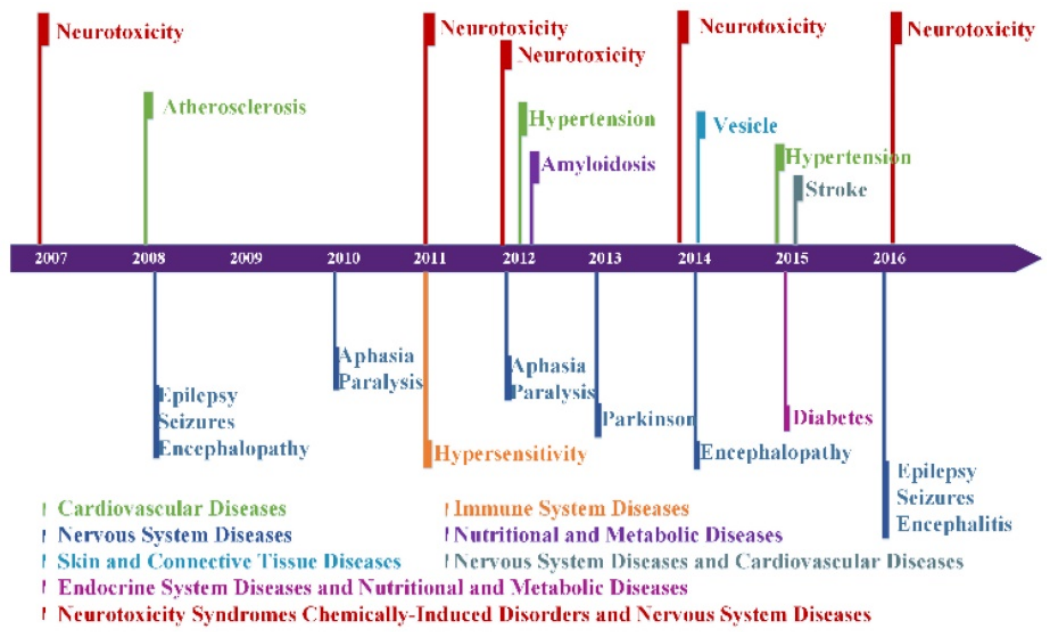

Figure 4. Disease associations with AD in each year. Different colors represent different categories of diseases. Neurotoxicity, Stroke, and Diabetes belong to two categories of diseases at the same time.

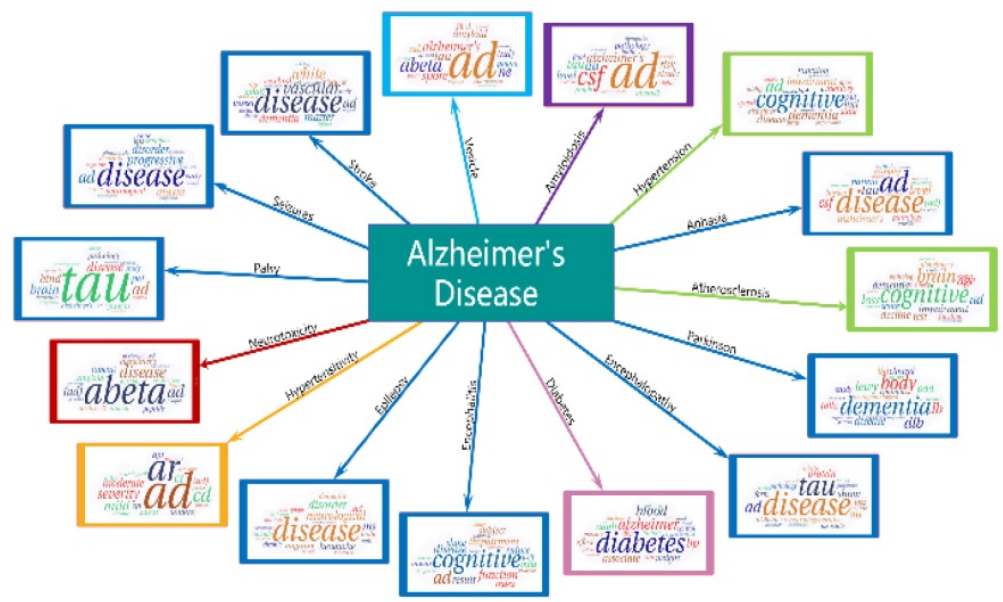

Figure 5. AD emerges in 15 diseases hotspots. The arrow indicates different diseases and the word clouds are the hotspots of them.

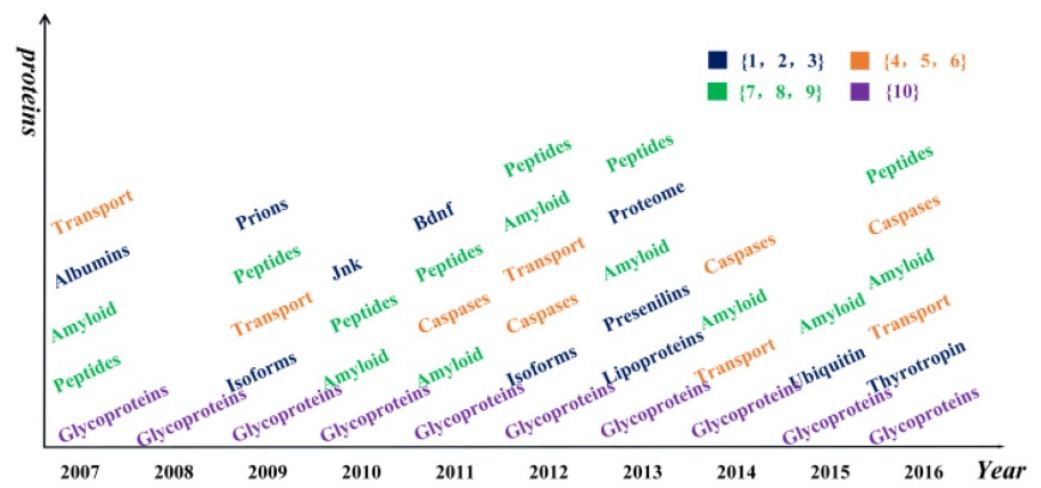

Figure 6. Hotspot words on protein in each year. Different colors represent the frequencies of different kinds of proteins. The numbers in brace are the frequencies of proteins appeared.

Glycoproteins appeared in all years, and amyloid appeared in 8 years from 2007 to 2016, except for 2008 and 2009. It is well known that amyloid and glycoprotein are important to AD [36, 37]. Apart from these words, some chemicals and compounds were found in the results, such as analgesics, estrogens, glucocorticoids, statin, and protease inhibitors, as listed in Table 2. We found the study of chemicals did not appear in 2015. And the study of the organic chemical was concentrated from 2008 to 2010. 


\section{Symptoms and phenomena hotspots}

It is well known that $\mathrm{AD}$ patients have difficulty in remembering recent events and have problems with language. However, other symptoms and phenomena are also common, such as aphasia and telomere. The complete list of symptoms and phenomena from our data is shown in Figure 7.

Table 2. Occurrence of chemicals and compounds

\begin{tabular}{lll}
\hline \multirow{2}{*}{ Word } & Categories Appearing in Each Year & \\
\cline { 2 - 3 } donepezil & Categories & Year \\
\hline & $\begin{array}{l}\text { Heterocyclic compounds; Polycyclic } \\
\text { compounds; Organic chemicals }\end{array}$ & 2009 \\
galantamine & Heterocyclic compounds & 2009 \\
stemazole & $\begin{array}{l}\text { Heterocyclic compounds; Organic } \\
\text { chemicals }\end{array}$ & 2011 \\
& Heterocyclic compounds; Polycyclic & 2014 \\
rifampicin & compounds & \\
serotonin & Heterocyclic compounds; Organic & 2010,2016 \\
& chemicals & \\
progesterone & Polycyclic compounds & 2007 \\
matrix & Polycyclic compounds & 2008 \\
oxysterols & Polycyclic compounds & 2008 \\
testosterone & Polycyclic compounds & 2016 \\
estradiol & Polycyclic compounds & 2016 \\
cholesterol & Polycyclic compounds & 2013 \\
carnitine & Organic chemicals & 2008 \\
ceramides & Organic chemicals & 2008 \\
isoflurane & Organic chemicals & 2008 \\
lactate & Organic chemicals & 2008 \\
acetylcholine & Organic chemicals & 2009 \\
ginsenosides & Organic chemicals & 2009 \\
choline & Organic chemicals & 2010 \\
inositol & Organic chemicals & 2010 \\
sulforaphane & Organic chemicals & 2010 \\
\hline
\end{tabular}

Apart from the decline of memory, the cognition, attention, memory recall, learning and speech abilities of AD patients are also declined. In addition, inflammation, neuroprotection, telomere, syndrome, synapsis also appeared, which could potentially suggest a composite screening or early warning of Alzheimer's disease based on these symptoms.

\section{Gene pathway hotspots}

We identified all the genes appeared in the topics, and 556 genes were found in total. To analyze these genes, we uploaded these genes to the David database [38] to find the enriched pathways of these genes.

As a result, 12 pathways were achieved; two of them are the Alzheimer's disease pathway and the nitrogen metabolism pathway. In the $\mathrm{AD}^{\prime} \mathrm{s}$ pathway, 12 genes were taken from our results, including the ADAM metallopeptidase domain 10 (ADAM10), LDL receptor related protein 1 (LRP1), amyloid beta precursor protein (APP), apolipoprotein $\mathrm{E}$ (APOE), beta-secretase 1 (BACE1), cyclin dependent kinase 5 (CDK5), microtubule associated protein tau (MAPT), insulin degrading enzyme (IDE), presenilin 1 (PSEN1), presenilin 2 (PSEN2), synuclein alpha (SNCA) and tumor necrosis factor (TNF). The P-value of the pathway is $4.20 \mathrm{E}-07$, and the false discovery rate (FDR) is 5.08E-04 according to our uploaded genes. Both P-value and FDR are less than 0.01, indicating that the pathway of our uploaded genes is statistically significant.

The pathway of Alzheimer's disease is shown in Figure 8 , and the genes in our results are marked by red stars to distinguish them with other genes. The original source of this pathway is from the KEGG website [39, 40]. The other 11 pathways are shown in Supplemental Table S4 and Figure S10-S20.

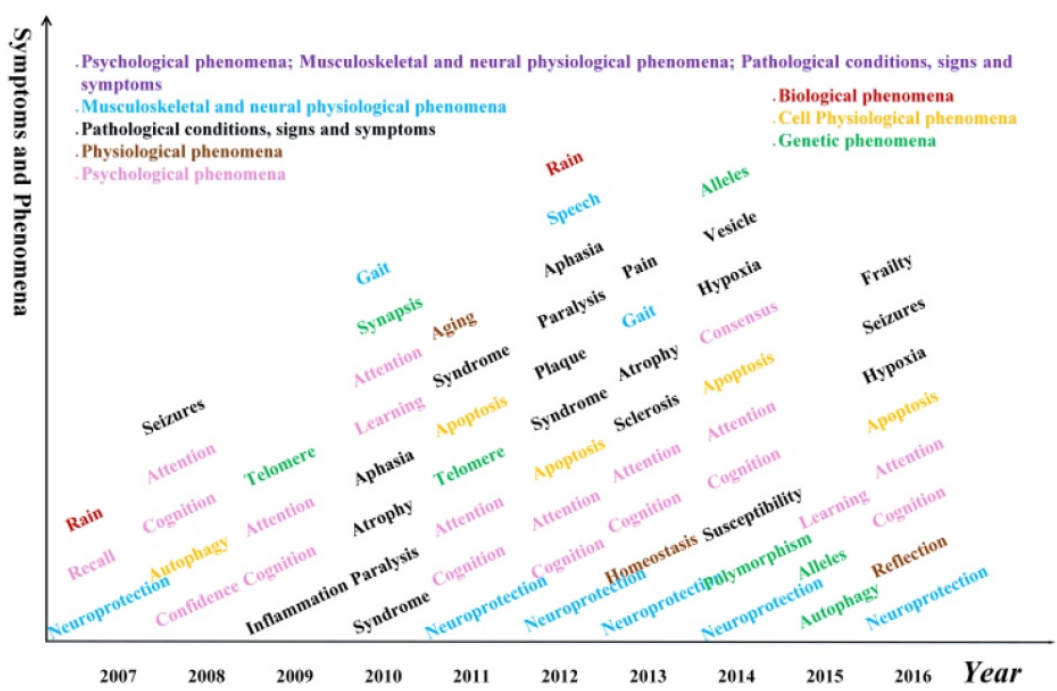

Figure 7. Hotspot words on symptom and phenomena in each year. Different colors represent different categories of symptoms or phenomena. 


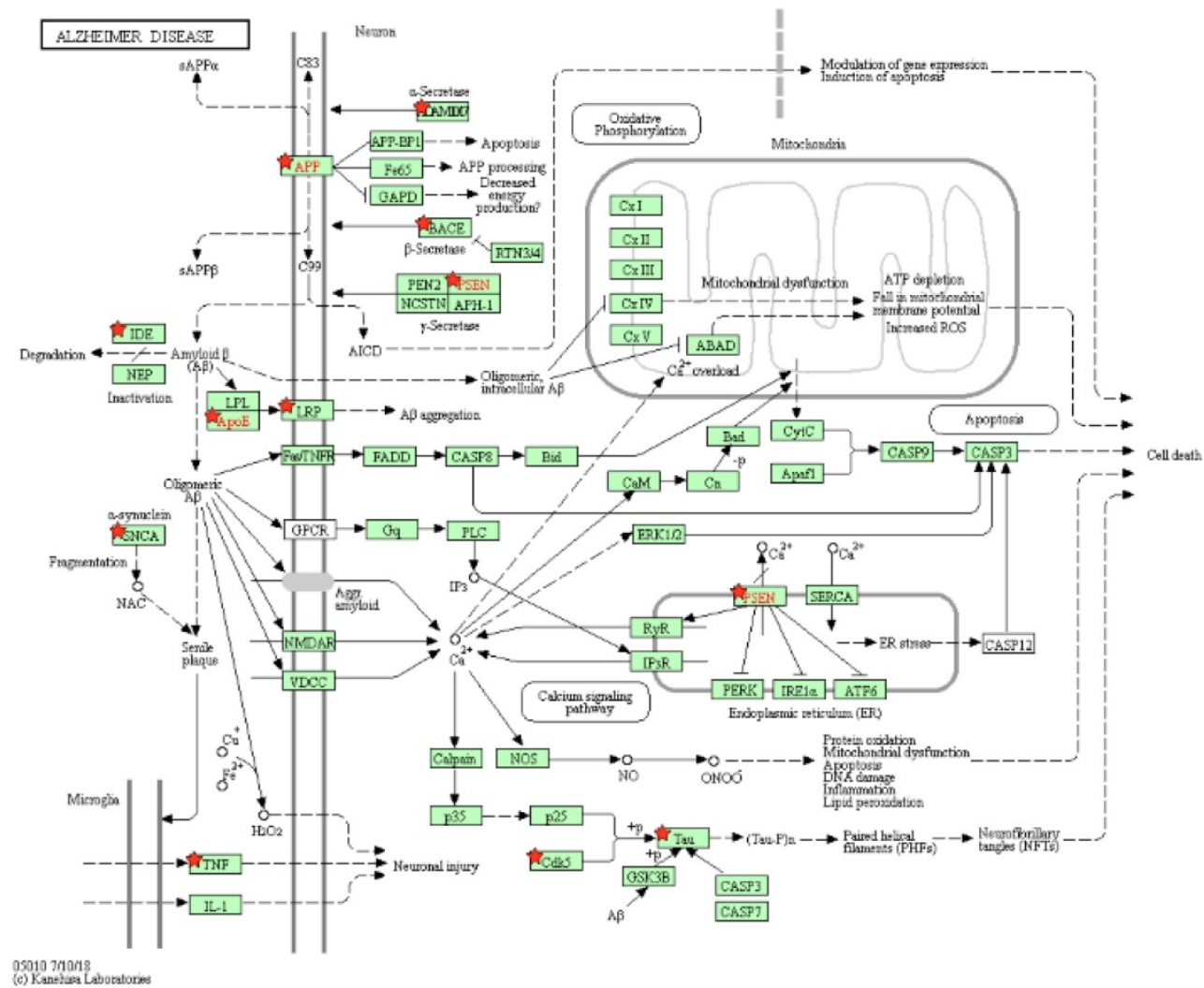

Figure 8. Pathway of Alzheimer's disease. Our uploaded genes are marked by red stars. The red genes are labeled by KEGG, and they are in our results.
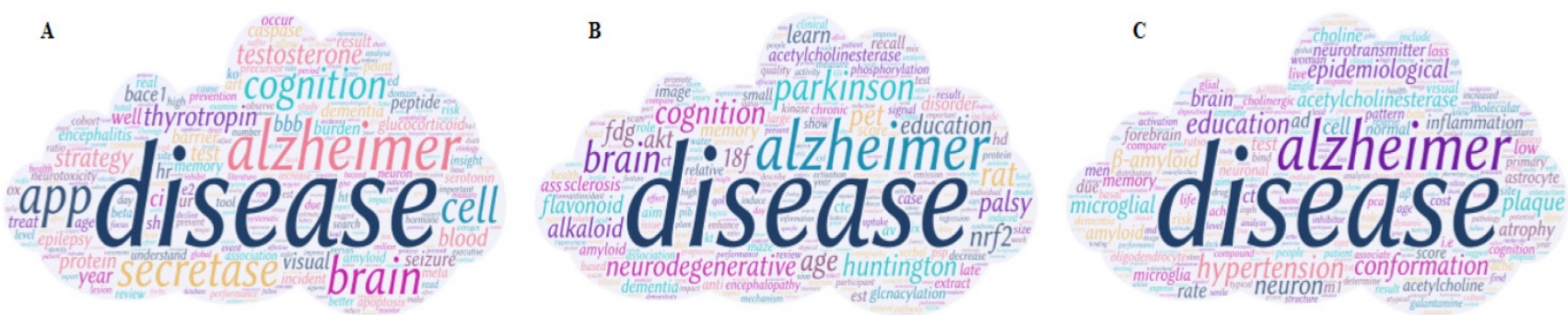

Figure 9. Key topics of AD from 2016 to 2018. (A) 2016 key topics. (B) 2017 key topics. (C) 2018 key topics. Different size of words displays the word's weight. The higher the word weight is, the bigger the word is.

\section{AD research trends}

We analyzed the trends and hotspots on center topics and three other aspects based on center topic words. AD research continues to evolve year after year, and there are some latent tendencies in the past years. To capture these tendencies, we merged all the central topics of the whole year into a word cloud. Taking 2016 as an example, the visualization of the result is shown in Figure 9A. Alzheimer, disease, time, brain, app, cost, and cell are the most noticeable words represented the research hotspots in 2016. The other figures from 2007 to 2015 are shown in the supplemental material as S21-S29.

Combined with the results of the 10-year (from 2007 to 2016) word clouds, we can infer that (1) phenomena, amino acids, peptides, and proteins appeared in each year and the disease category appeared in 9 years (except 2009). These categories are considered as long-term research hotspots and directly related to AD. Therefore, we can predict that these categories would be the hotspots topics in 2017 and 2018. To validate the prediction, we generated AD-research hotspots in 2017 and 2018 using LDAP and achieved the validation. We found these three categories in Figure 9B and 9C, where disease category appeared on the upper part of the word clouds, as Parkinson, Huntington, palsy, Neurodegenerative Diseases, encephalopathy, and sclerosis appear in 2017 and hypertension appears in 2018. (2) From Figure 4, we find that hypertension occurs in 2012 and 2015 with a time interval of 3 three years, therefore, we predict that it will appear in 2018. In the result of 2018 (Figure 9C), we found it in pink under the "disease" topic.

In addition, the proportion of MeSH category 
about the hotspots from 2007 to 2018 is shown in Figure 9. Different colors represent the proportion of different types of research in a year. In Figure 10, the hormone category appeared in 2007 and 2016. In 2016, the proportion of hormones was twice that of 2007. It implies that the research on hormone has made new discoveries for AD around 2016. We found clues from the researches on whether the growth hormone can be contaminated by amyloid- $\beta$ seeds as well as by prions, which were published in Nature in September 2015 and November 2016 [41, 42]. Therefore, we also find the hormone category in 2017. These evidences certified our research trends results.

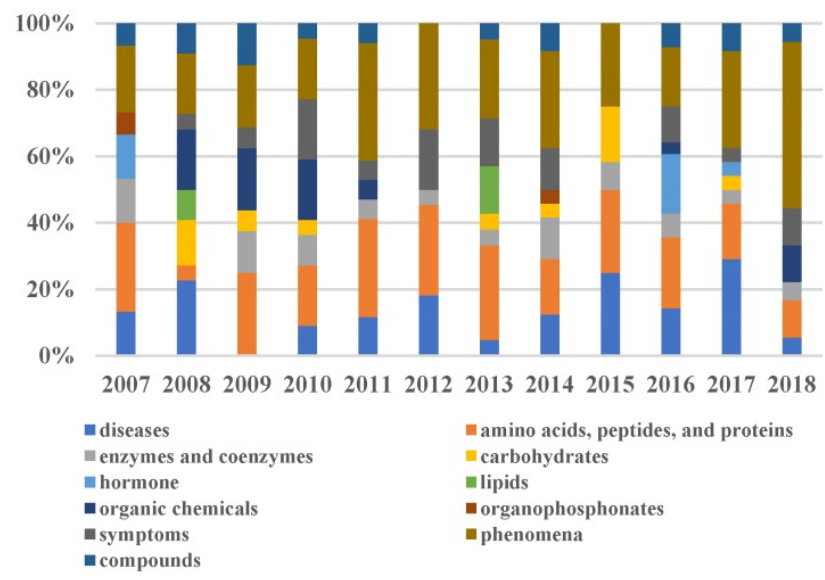

Figure 10. Proportion of each category about the hotspots from 2007 to 2018. Different colors represent different categories. The height of each color indicates the proportion of that category.

\section{Conclusions}

To reveal the hotspots and trends of $\mathrm{AD}$, we proposed a novel LDAP framework which combines topics model and AP algorithm. From about 100,000 $\mathrm{AD}$ papers, we found the spotlights of $\mathrm{AD}$ research including 556 genes, 15 diseases, 15 amino acids, peptides, and proteins, 19 chemicals and compounds, 33 symptoms and phenomena, and 12 related pathways. We studied the research hotspots via a visualization method to find the regularities, and reveal the research hotspots on $\mathrm{AD}$ research. It should be noted that there is no unique criteria to explain the trends of $\mathrm{AD}$. In this paper, we mainly use the information of PubMed abstracts through machine learning models. This may have some bias for completely understanding the trends of $\mathrm{AD}$. However, the discovery of the research trends and hotspots evolution on AD are supposed to provide some guidance for further research and might to useful to drug study. For example, a drug used to treat a disease associated with the 556 AD related genes may treat or alleviate some of the symptoms of AD. In addition, the 15 amino acids, peptides, and proteins, 9 enzymes and coenzymes, 7 hormones, 7 carbohydrates, 5 lipids, 2 organophosphonates, 18 chemicals, 11 compounds can be made into corresponding inhibitors, which may be used to treat AD. These results are required further experimental verification. In addition to the research manuscripts, patents about $\mathrm{AD}$ also can provide the hotspots from different views, especially for the drug development. We will introduce this data in future research.

\section{Supplementary Material}

Supplementary figures and tables. http://www.ijbs.com/v15p2065s1.pdf

\section{Acknowledgments}

The authors are grateful for the support of the National Natural Science Foundation of China (61602207 and 61572228), the Science Technology Development Project from Jilin Province (20190302107GX), Special Research and Development of Industrial Technology of Jilin Province (2019C053-7), Guangdong Key-Project for Applied Fundamental Research (2018KZDXM076), and Guangdong Premier Key-Discipline Enhancement Scheme (2016GDYSZDXK036). This work was also partially supported by the US National Institutes of Health grant R35-GM126985.

\section{Competing Interests}

The authors have declared that no competing interest exists.

\section{References}

1. Brookmeyer R, Johnson E, Ziegler-Graham K, Arrighi HM. Forecasting the global burden of Alzheimer's disease. Alzheimer's \& Dementia. 2007; 3(3):186-91.

2. [Internet] Prince MJ. World Alzheimer Report 2015: The Global Impact of $\begin{array}{lllll}\text { Dementia. } & 2015 . & \text { Revised } & 25 & \text { February }\end{array}$ https://www.alz.co.uk/research/world-report-2015

3. Vos T, Allen $\mathrm{C}$, Arora $\mathrm{M}$, et al. Global, regional, and national incidence, prevalence, and years lived with disability for 310 diseases and injuries, 1990-2015: a systematic analysis for the Global Burden of Disease Study 2015. The Lancet. 2016; 388(10053):1545-1602.

4. Weuve J, Hebert LE, Scherr PA, Evans DA. Deaths in the United States among persons with Alzheimer's disease (2010-2050). Alzheimer's \& Dementia. 2014; 10(2):e40-46.

5. Bengt W, Philippe A, Sandrine A, et al. Defeating Alzheimer's disease and other dementias: a priority for European science and society. The Lancet Neurology. 2016; 15(5):455-532.

6. Lobo A, Launer LJ, Fratiglioni L, Andersen K, Di Carlo A, Breteler MMB, et al. Prevalence of dementia and major subtypes in Europe: a collaborative study of population-based cohorts. Neurology. 2000; 54(Suppl 5):S4-S9.

7. [Internet] Dementia. World Health Organization. Revised 2 September 2018. http://www.who.int/news-room/fact-sheets/detail/dementia

8. [Internet] G8 dementia summit agreements. GOV.UK. Revised 2 September 2018.

https://www.gov.uk/government/publications/g8-dementia-summit-agree ments

9. Wang $\mathrm{H}$, Naghavi $\mathrm{M}$, Allen $\mathrm{C}$, et al. Global, regional, and national life expectancy, all-cause mortality, and cause-specific mortality for 249 causes of death, 1980-2015: a systematic analysis for the Global Burden of Disease Study 2015. The Lancet. 2016; 388(10053):1459-1544.

10. Norton S, Matthews FE, Brayne C. A commentary on studies presenting projections of the future prevalence of dementia. BMC Public Health. 2013; 13(1):1. 
11. Ferri CP, Prince M, Brayne C, Brodaty H, Fratiglioni L, Ganguli M, et al. Global prevalence of dementia: a Delphi consensus study. The Lancet. 2005; 366(9503):2112-2117.

12. Alzheimer's Association. 2018 Alzheimer's disease facts and figures. Alzheimer's \& Dementia. 2018; 14(3):367-429.

13. Mendez MF. Early-onset Alzheimer's disease: nonamnestic subtypes and type 2 AD. Arch Med Res. 2012; 43(8):677-685.

14. Burns A, Iliffe S. Alzheimer's disease. BMJ. 2009; 338:b158.

15. Alzheimer's Association. 2019 Alzheimer's disease facts and figures. Alzheimer's \& Dementia. 2019; 15(3):321-387.

16. [Internet] What Are the Signs of Alzheimer's Disease? National Institute on $\begin{array}{llll}\text { Aging. } & \text { Revised } & 2 & \text { September } \\ \end{array}$ http://www.nia.nih.gov/health/what-are-signs-alzheimers-disease

17. Querfurth HW, LaFerla FM. Alzheimer's Disease. New England Journal of Medicine. 2010; 362(4):329-344.

18. Todd S, Barr S, Roberts M, Passmore AP. Survival in dementia and predictors of mortality: a review. International Journal of Geriatric Psychiatry. 2013; 28(11):1109-1124.

19. [Internet] Alzheimer's Association. Alzheimer's Disease \& Dementia Help. Revised 2 June 2019. https:/ / www.alz.org/

20. Honig LS, Vellas B, Woodward M, et al. Trial of Solanezumab for Mild Dementia Due to Alzheimer's Disease. New England Journal of Medicine. 2018; 378(4):321-330.

21. Hsu DA. Marshall G. Primary and Secondary Prevention Trials in Alzheimer Disease: Looking Back, Moving Forward. Current ALzheimer Research. 2017; 14(4):426-440.

22. Bonin-Guillaume S, Zekry D, Giacobini E, Gold G, Michel JP. The economical impact of dementia. Presse Med. 2005; 34(1):35-41.

23. Meek PD, McKeithan EK, Schumock GT. Economic Considerations in Alzheimer's Disease. Pharmacotherapy. 1998; 18(2P2):68-73.

24. Feng X, Zhang $\mathrm{H}$, Ren $\mathrm{Y}$, et al. The Deep Learning-Based Recommender System "Pubmender" for Choosing a Biomedical Publication Venue: Development and Validation Study. J Med Internet Res. 2019; 21(5):e12957.

25. Blei DM, Lafferty JD. A correlated topic model of Science. Ann Appl Stat. 2007; 1(1):17-35.

26. Blei DM, Ng AY, Jordan MI. Latent Dirichlet Allocation. Journal of Machine Learning Research. 2003; 3:993-1022.

27. Wei X, Croft WB. LDA-based Document Models for Ad-hoc Retrieval. In: Proceedings of the 29th Annual International ACM SIGIR Conference on Research and Development in Information Retrieval. New York, ACM; 2006: 178-185.

28. Hennig L, Tu DL. Topic-based multi-document summarization with probabilistic latent semantic analysis. In: Proceedings of the International Conference RANLP-2009. Bulgaria: Borovets; 2009: 144-149.

29. Frey BJ, Dueck D. Clustering by Passing Messages Between Data Points. Science. 2007; 315(5814):972-976.

30. Yang C, Bruzzone L, Sun F, et al. A Fuzzy-Statistics-Based Affinity Propagation Technique for Clustering in Multispectral Images. IEEE Transactions on Geoscience and Remote Sensing. 2010; 48(6):2647-2659.

31. Guan R, Shi X, Marchese M, et al. Text Clustering with Seeds Affinity Propagation. IEEE Transactions on Knowledge and Data Engineering. 2011; 23(4):627-637.

32. Leone $\mathrm{M}$, et al. Clustering by soft-constraint affinity propagation: applications to gene-expression data. Bioinformatics. 2007; 23(20):2708-2715.

33. [Internet] Good Elementary School Grades Linked to Lower Dementia Risk. $\begin{array}{lllll}\text { Medscape. } & \text { Revised } & 22 & \text { February } & 2019 .\end{array}$ http://www.medscape.com/viewarticle/848332

34. American Diabetes Association. Standards of medical care in diabetes-2016. Diabetes Care. 2016; 39(Suppl 1):S1-S112.

35. He G, Liang Y, Chen Y, et al. A hotspots analysis-relation discovery representation model for revealing diabetes mellitus and obesity. BMC Systems Biology. 2018; 12(Suppl 7):116.

36. Castello MA, Soriano S. On the origin of Alzheimer's disease. Trials and tribulations of the amyloid hypothesis. Ageing research reviews. 2014; 13:10-12.

37. Budge KM, Neal ML, Richardson JR, Safadi FF. Glycoprotein NMB: an Emerging Role in Neurodegenerative Disease. Mol Neurobiol. 2018; 55(6):5167-5176.

38. [Internet] DAVID Functional Annotation Bioinformatics Microarray Analysis. Revised 22 February 2019. https:/ / david.ncifcrf.gov/

39. [Internet] KEGG: Kyoto Encyclopedia of Genes and Genomes. Revised 22 February 2019. https://www.genome.jp/kegg/

40. Kanehisa, Furumichi M, Tanabe M, et al. KEGG: new perspectives on genomes, pathways, diseases and drugs. Nucleic Acids Res. 2017; 45(D1):D353-D361.

41. Jaunmuktane Z, Mead S, Ellis M, et al. Evidence for human transmission of amyloid- $\beta$ pathology and cerebral amyloid angiopathy. Nature. 2015; 525:247-250.

42. Collinge J. Mammalian prions and their wider relevance in neurodegenerative diseases. Nature. 2016; 539:217-226. 